\title{
CALIDAD DE VIDA EN UN GRUPO DE PACIENTES CON DIABETES MELLITUS TIPO 2: UN ESTUDIO TRANSVERSAL
}

\author{
Rojas Giovanni ${ }^{1}$, ${ }^{2 *}$, Solís Pazmiño Paola ${ }^{3}$, Gaona Richard ${ }^{1}$, Mollocana Fernanda ${ }^{1}$, \\ Espín Ismael ${ }^{1}$, Nunes Altacílio ${ }^{2}$
}

DOI: $10.48018 /$ rmv.v32.i1.7

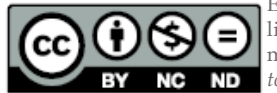

Este artículo está bajo un licencia de Creative Commons de tipo Reconocimien OPEN ACCESS to - No comercial - Sin obras

1. Universidad Central del Ecuador. Facultad de Ciencias Médicas. Escuela de Medicina. Quito - Ecuador. 2. Universidade de São Paulo. Faculdade de Medicin de Riberão Preto. Departamento de Medicina Social. Riberão Preto - Brasil.

3. Stanford University. Department of Otolaryngology and Head and Neck Surgery

Stanford, California - United States of America.

\section{ORCID ID:}

Rojas Giovanni

orcid.org/0000-0002-9117-9223

Solís Pazmiño Paola

orcid.org/0000-0003-1725-232X

Gaona Richard

orcid.org/0000-0002-4006-6552

Mollocana Fernanda

orcid.org/0000-0001-5916-4403

Espín Ismael

orcid.org/0000-0002-5004-3709

Nunes Altacílio

orcid.org/0000-0001-9934-920X

*Corresponding author: Giovanni Rojas

E-mail: gwrojas@uce.edu.ec

Article history

Received: 13 - Dic - 2020

Accepted: 10 - May - 2021

Publish: 01 - Jun - 2021

STROBE 2008 Check List statement: The authors have real the STROBE 2008 Check List and the manuscript was prepared and revised according to the STROBE 2008 Checklist.

Conflict of interest: All authors declared that there are no conflicts of interest.

Financial disclosure: The authors have no financial relationships relevant to this article to disclose.

\section{Authors' contribution:}

Conception and/or delineation of the study: GR, AN data collection: GR, PS, RG, FM, IE; statistical analysis: GR, PS, AN; analysis and interpretation of data: GR, AN PS, RG; preliminary drafting: GR, PS; critical preview review: AN, PS, RG, FM, IE.

All the authors reviewed and approved the final manuscript.

Citation: Rojas G, Solís Pazmiño P, Gaona R, Mollocana F,

Espín I, Nunes A. CALIDAD DE VIDA EN UN GRUPO DE PACIENTES CON DIABETES MELLITUS TIPO 2: UN ESTUDIO TRANSVERSAL. Rev Med Vozandes. $2021 ; 32$ (1): 13-22

\section{Resumen}

\section{Introducción}

En la toma de decisiones en salud se debe dar relevancia a la percepción del paciente sobre la calidad de vida derivada del estado de salud resultante del uso de una $u$ otra alternativa posible. A nivel mundial, la utilidad asociada a la calidad de vida se expresa en años de vida ajustados por calidad (AVAC ○ QALY). El EQ-5D-5L ha mostrado validez y fiabilidad como medida de calidad de vida y utilidad en salud, siendo estudiado y validado tanto en la atención primaria como en pacientes con diabetes.

\section{Objetivo}

Cuantificar la calidad de vida percibida por los pacientes con diabetes mellitus tipo 2 durante el año 2018 en unidades de utilidad, empleando el cuestionario EQ-5D-5LTM.

\section{Pacientes y métodos}

Estudio transversal con participantes adultos con más de 40 años, diabetes mellitus tipo 2 en tratamiento y que asisten a la consulta externa. LOS QALY se estimaron con la calculadora del sitio web NATSINC. Los resultados se expresaron en medias con desviación estándar o porcentajes, y se realizaron medidas de asociación entre variables.

\section{Resultados}

57 participantes principalmente mujeres cumplieron los criterios de inclusión y exclusión, con una media de edad de 67.77 años. El estado de salud más frecuente fue el 11111 (28.1\%) que significa "ningún malestar". Se obtuvo una media de 74.47 en la EVA y de 0.708 en QALY.

\section{Conclusiones}

Se reporta un mayor nivel de calidad de vida en comparación a países con niveles económicos superiores, con promedios muy similares en los puntajes obtenidos en la EVA.

Palabras clave: calidad de vida, QALY, diabetes mellitus tipo 2 
Keywords: quality of life, QALY, diabetes mellitus type 2

\section{Abstract}

\section{QUALITY OF LIFE IN A GROUP OF PATIENTS WITH TYPE 2 DIABETES MELLITUS: A CROSS-SECTIONAL STUDY}

\begin{abstract}
Introduction
In health decision-making, relevance should be given to the patient's perception of the quality of life derived from the state of health resulting from the use of one or another possible alternative. Worldwide, the utility associated with quality of life is expressed in quality-adjusted life years (QALY). The EQ-5D-5L has shown validity and reliability as a measure of quality of life and utility in health, being studied and validated both in primary care and in diabetic patients.
\end{abstract}

Objective

To measure the perceived quality of life in patients with diabetes mellitus type 2 in utility measures, in a second-level hospital during 2018 using the EQ-5D-5L questionnaire Spanish version for Ecuador.

\begin{abstract}
Patients and methods
Cross-sectional study with adults older than 40 years with type 2 diabetes mellitus in treatment and ambulatory medical assistant. The QALYs were estimated using the calculator on the NATSINC website. The results were expressed in means with standard deviation or percentages, and measures of association between variables were performed.
\end{abstract}

Results

57 participants, mainly women, fulfilled the inclusion and exclusion criteria; with an average age of 67.77 years. Regarding the quality of life measured by the EQ-5D-5L instrument, the most frequent state of health was 11111 (28.1\%), which means "no discomfort". A mean of 74.47 was obtained in the EVA and 0.708 in QALY.

\section{Conclusions}

The participants in this study report a higher level of quality of life compared to countries with higher economic levels, and very similar averages in the scores obtained in the EVA.

\section{Introducción}

Referirse al concepto de calidad es en ocasiones complicado pues se trata de una propiedad muchas veces intangible de personas, instituciones o procesos, y susceptible de múltiples interpretaciones. Su introducción desde el mundo económico al campo de la salud ha buscado la mejora continua de las prestaciones y servicios que se brindan. (1) Existen múltiples definiciones de calidad de las cuales citaremos la del Instituto de Medicina Americano que en 1974 estableció que "la atención sanitaria de calidad es aquella que es efectiva en la mejora del nivel de salud y grado de satisfacción de la población, con los recursos que la sociedad y los individuos han decidido destinarle" (2).

A partir de los años noventa del siglo veinte para la toma de decisiones en salud se empieza a dar relevancia a la percepción del paciente sobre la calidad de vida derivada del estado de salud resultante del uso de una u otra alternativa posible. Así el concepto de calidad de vida hace referencia a la "valoración subjetiva que un individuo hace sobre el nivel de bienestar que percibe, de acuerdo con sus propias condiciones físicas, mentales, emocionales y sociales en determinado momento". Por lo que esta valoración se ve afectada por factores medioambientales, socioculturales, y por cómo el paciente interactúa con su entorno. ${ }^{(3)}$

Sin embargo, dado que el concepto de calidad de vida abarca aspectos relacionados y no 
relacionados con la salud se puede hablar de manera más específica de la calidad de vida relacionada con la salud (CVRS o health-related quality of life - HRQOL). Este nuevo concepto "representa el efecto funcional de una enfermedad y su consecuente terapia sobre un paciente tal como este lo percibe". Además del componente físico, la CVRS incluye otros aspectos de la salud importantes para el paciente llamados dominios, como pueden ser su funcionamiento social y psicológico. ${ }^{(4)}$

Para cuantificar la CVRS, varios autores utilizan los términos "utilidad", "valor" y "preferencia" de manera intercambiable; sin embargo, hacen referencia a aspectos diferentes. Preferencia abarca tanto a la utilidad como al valor ${ }^{(5)}$ y utilidad hace referencia "a la calidad percibida o al deseo relativo de los diferentes sujetos entre dos o más estados de salud". (6) Su definición en evaluaciones económicas en salud nace de la aplicación de los trabajos sobre la teoría de juegos de von Neumann y Morgestern (1944), quienes definieron a la utilidad como la "preferencia explícita y cuantitativa (valor) expresada por un tomador de decisiones, frente a un desenlace específico". ${ }^{(7)}$

Estos estados de salud pueden medirse mediante técnicas que cuantifican su utilidad de manera directa o indirecta, o mediante medidas multidimensionales de la calidad de vida asociada a salud (llamadas medidas de no utilidad o no preferencia). Las medidas de utilidad tienen como punto de vista a la sociedad para cuantificar la preferencia, y las de no utilidad se centran en el paciente. ${ }^{(4)}$ Técnicas para obtener medidas de utilidad de manera directa son: a) la escala de categorías (rating scale) y la escala visual analógica (EVA o visual analogue scale - VAS -), b) la equivalencia temporal (time tradeoff -TTO), y c) la lotería estándar o apuesta normalizada (standard gamble): mientras que técnicas indirectas corresponden a sistemas genéricos de clasificación como los instrumentos SF-6D, EQ$5 \mathrm{D}$ y HUl-3. Estas cuantificaciones de utilidad brindan una medida de la preferencia relativa por un efecto o estado de salud concreto resumiendo todos los aspectos positivos y negativos de la calidad de vida tal y como lo vive y siente el propio sujeto, una vez que han sido incorporadas actitudes respecto al riesgo y la duración de la vida. ${ }^{(6)}$ A nivel mundial la utilidad asociada a la calidad de vida se expresa en años de vida ajustados por calidad (AVAC O QALY- quality adjusted life year -), el cual se entiende como una medida de resultados en salud que considera la cantidad y calidad de vida en un único valor donde un año de vida en perfecta salud equivale a 1 QALY-AVAC y la muerte valdría cero. ${ }^{(8)}$

El instrumento EQ-5D nació entre 1987 y 1990 de la iniciativa multidisciplinaria europea EroQol, la cual buscaba crear un índice en salud que combinara la cantidad y la calidad de vida para ser utilizado en evaluaciones económicas como una medida de efectividad. Esteinstrumento genérico está integrado por las cinco dimensiones consideradas más relevantes de la CVRS: movilidad, autocuidado, actividades habituales, dolor/ malestar y ansiedad/depresión; con tres niveles de gravedad en cada dimensión: ausencia de problema, algún problema, problema grave o incapacidad). El instrumento está formado por dos partes: el sistema descriptivo EQ-5D y la escala visual analógica (EVA) según el cual los entrevistados valoran distintos estados de salud en una escala con valores de 0 a 100. Es un cuestionario genérico y estandarizado, autoadministrado, fácil de responder y con poca carga cognitiva. Su uso se ha generalizado en todo el mundo y está disponible en más de 170 idiomas, incluidas varias versiones en español para distintos países tras un proceso riguroso de traducción. ${ }^{(9,10)}$

El EQ-5D ha mostrado su validez y fiabilidad como medida de utilidad en salud; sin embargo, su versión original tenía limitaciones como un efecto techo y bajo poder discriminatorio especialmente en los cambios pequeños en los estados de salud más leves. Con la finalidad de superar estos problemas, en 2009 se lanzó la versión EQ-5D-5L añadiendo dos niveles en cada una de las dimensiones (sin problemas, problemas leves, problemas moderados, problemas graves y problemas extremos/imposibilidad), con lo que se definen un total de $3125^{\left(5^{5}\right)}$ estados de salud. El EQ-5D-5L también ha demostrado ser una extensión válida del EQ-5D-3L mejorando sus propiedades de medición y está disponible en más de 120 idiomas; inclusive con una versión juvenil llamada EQ-5D-Y. ${ }^{(10)}$

Adicionalmente, la versión EQ-5D-5L ha sido estudiada y validada para cuantificar la calidad de vida de pacientes tanto en la atención primaria en general ${ }^{(11,12)}$ como de manera específica en pacientes con diabetes $\left({ }^{13,14)}\right.$. En relación con otros instrumentos como el SF-6D y DHP-18, ha mostrado ser el más adecuado para determinar la calidad de vida asociada al estado de salud ${ }^{(5,15)}$. De esta manera el objetivo principal de este estudio fue cuantificar la calidad de vida percibida por los pacientes con diabetes mellitus tipo 2 durante el año 2018 en unidades de utilidad, empleando el cuestionario EQ-5D-5L ${ }^{\mathrm{TM}}$ versión en español para Ecuador.

\section{Pacientes y Métodos}

\section{Diseño de estudio}

Se realizó un estudio transversal en pacientes con diagnóstico de diabetes mellitus tipo 2 que asistieron a consulta externa de Medicina Familiar y/o Medicina Interna del Hospital Un Canto a la Vida (unidad de salud de segundo nivel), entre enero a julio del 2018. Para la presentación de los resultados se siguieron las recomendaciones de la iniciativaSTROBE ${ }^{(16)}$, así comolas recomendaciones del EuroQol Group ${ }^{\mathrm{TM}}$ (9)

El estudio forma parte de un proyecto de investigación para realizar un análisis farmacoeconómico del uso de sitagliptina en los pacientes atendidos en el hospital base, utilizando medidas de utilidad y modelos de predicción. 


\section{Número y selección de los participantes en el estudio}

Por el pequeño número de pacientes atendidos en la institución con el diagnóstico de diabetes mellitus tipo 2 durante el año previo a este estudio (39 pacientes sin mención de complicaciones y 67 con alguna complicación), se buscó trabajar con el mayor número posible de individuos sin realizarse cálculo muestral.

Los pacientes fueron identificados mientras esperaban su atención médica en la consulta externa del Hospital, donde se les invitaba a participar en el estudio. Aquellos que aceptaron y cumplieron los criterios de inclusión (adultos de 40 años de edad o más con el diagnóstico de diabetes mellitus tipo 2, de ambos sexos, atendidos en consulta externa de Medicina Interna o Medicina Familiar, que aceptaron participar y capaces de leer y escribir) y exclusión (menores de 40 años, otros tipos de diabetes, demencia o complicaciones neurológicas poreventos cerebrovasculares, ceguera, amputación de extremidades e insuficiencia renal crónica terminal en hemodiálisis); firmaron el respectivo consentimiento informado y se les aplicó los dos instrumentos de este estudio por algún miembro del equipo de investigación debidamente capacitado (EQ-5D-5 $\mathrm{L}^{\mathrm{TM}}$ y cuestionario con variables sociodemográficas y económicas).

\section{Variables sociodemográficas y económicas}

El cuestionario de preguntas sociodemográficas hacía referencia a la edad, sexo, estado civil, nivel de escolaridad, ocupación, ingresos económicos, así como sobre otras comorbilidades que presenten al momento de su evaluación. Esto se realizó considerando el papel de estos factores sobre la calidad de vida.

\section{Análisis estadístico}

La información de los cuestionarios fue introducida en la base de datos desarrollada especialmente para el estudio en la plataforma de Microsoft Excel ${ }^{\circledR}$. Los cuestionarios que no estuvieron completamente llenos o con respuestas en blanco fueron ignorados. Las variables cualitativas se midieron con porcentajes, y las variables cuantitativas con media y desviación estándar como medidas de tendencia central.

Las posibles asociaciones entre variables cualitativas se estimaron con la prueba de chi-cuadrado. Para comparar las variables cuantitativas se utilizó la prueba t de Student. Para todas las pruebas utilizadas, se consideró significancia estadística con un valor de $\mathrm{p}<0.05$ e intervalos de confianza al $95 \%$. Además, para las variables cuantitativas que mostraron asociación estadística significativa se calculó correlación de Pearson ( $r$ ) y coeficiente de determinación $\left(r^{2}\right)$. El análisis de los datos se realizó utilizando el programa SPSS ${ }^{\circledR}$ (versión 22).

La transformación de los estados de salud obtenidos con el instrumento EQ-5D-5LTM a medidas de utilidad (QALY-AVAC), se realizó utilizando la calculadora disponible en el sitio web del Núcleo de Evaluación de Tecnologías en Salud (NATSINC) ${ }^{(8)}$. Esta calculadora se basa en el estudio de Santos y colaboradores ${ }^{(18)}$, quienes emplearon la metodología TTO para obtener un algoritmo de cálculo de utilidades a través de la aplicación de un modelo de regresión de efectos mixtos a nivel individual sin ningún término de interacción.

\section{Consideraciones éticas}

El proyecto fue aprobado por el Comité de Ética de Investigación en Seres Humanos del Hospital Eugenio Espejo, por cuanto el hospital participante no cuenta con comité reconocido por el Ministerio de Salud Pública del Ecuador. Los pacientes no recibieron compensación económica alguna - algún otro estímulo que aliente de manera deliberada su participación. El manuscrito sigue las recomendaciones STROBE para estudios observacionales.

\section{Resultados}

Se lograron realizar 57 encuestas a pacientes que cumplieron los criterios de inclusión y exclusión; de los cuales 46 (80.7\%) fueron mujeres y 11 (19.3\%) hombres, con una media de edad de 67.77 años (ะ9.48) y un mínimo de 40 años y un máximo de 80 años. En cuanto a su estado civil, 35 (61.4\%) se encontraban casados. Los participantes en su mayoría contaban con escolaridad primaria (incompleta el $40.4 \%$ y completa el $29.8 \%$ ). 34 (59.6\%) participantes refirieron padecer de otra enfermedad además de la diabetes mellitus tipo 2, principalmente Hipertensión Arterial. En cuanto al antidiabético más utilizado por los pacientes, 29 (50.88\%) tomaban metformina, seguidos de la combinación de metformina con insulina 0 insulina sola, y metformina con un inhibidor de la dipeptidilpeptidasa-4 (iDPP-4). Estos datos se resumen en la tabla 1.

En relación con los datos económicos investigados, $38(66.7 \%)$ pacientes refirieron no trabajar, 28 (49.1\%) mencionaron percibir menos de una remuneración básica para el año de aprobación del estudio (equivalente a 375 dólares americanos), y donde sólo un miembro de la familia trabaja (63.2\% de encuestados). Estos datos se observan en la tabla 2.

En cuanto a las 5 dimensiones que componen el instrumento de calidad de vida EQ-5D$5 \mathrm{~L}, \quad 51$ (89.5\%) participantes no refirieron problemas en el cuidado y arreglo personal, 50 (87.7\%) no manifestaron problemas para realizar actividades cotidianas, 27 (47.4\%) no manifestaron padecer dolor al momento de la entrevista, y 31 (54.4\%) no expresaron angustia o depresión. Convirtiendo estos valores a la codificación de estados de salud se obtuvieron 25 estados, siendo el más frecuente el 11111 (equivale a "ningún malestar" en las cinco dimensiones) en 16 participantes (28.1\%). Estos datos se presentan en las tablas 3 y 4.

En cuanto a la salud en el día de la entrevista registrado mediante la EVA, se obtuvo una media de 74.47 ( \pm 18.339$)$, con valor mínimo de 40 y máximo de 100. El valor de cada QALY asociado a los estados de salud obtenidos en cada participante se obtuvo mediante la calculadora mencionada en la metodología, 
observándose un QALY promedio en este grupo de 0.708 $( \pm 0.276)$. En los nueve pacientes que utilizan tratamientos con insulina (sola o en combinación), se encontró un puntaje de EVA de 72.78 (similar al valor del grupo estudiado), y un QALY de 0,558 (menor al del grupo estudiado); datos que deben tomarse con precaución por el pequeño número de pacientes en tratamiento con insulina. No se realizaron otros análisis de subgrupos, dado que por el número pequeño de participantes los estadísticos sufrirían una mayor dispersión restando más significancia estadística.

\begin{tabular}{|c|c|c|}
\hline Variable & Frecuencia & Porcentaje \\
\hline \multicolumn{3}{|l|}{ Sexo } \\
\hline Mujer & 46 & $80.7 \%$ \\
\hline Hombre & 11 & $19.3 \%$ \\
\hline \multicolumn{3}{|l|}{ Estado civil } \\
\hline Casado & 35 & $61.4 \%$ \\
\hline Divorciado & 8 & $14 \%$ \\
\hline Viudo & 8 & $14 \%$ \\
\hline Soltero & 6 & $10.5 \%$ \\
\hline \multicolumn{3}{|l|}{ Instrucción } \\
\hline Primaria incompleta & 23 & $40.4 \%$ \\
\hline Primaria completa & 17 & $29.8 \%$ \\
\hline Secundaria incompleta & 7 & $12.3 \%$ \\
\hline Secundaria completa & 7 & $12.3 \%$ \\
\hline Superior & 3 & $5.3 \%$ \\
\hline \multicolumn{3}{|l|}{ Enfermedades adicionales } \\
\hline Sí & 34 & $59.6 \%$ \\
\hline No & 23 & $40.4 \%$ \\
\hline \multicolumn{3}{|l|}{ Tipo de enfermedad adicional } \\
\hline Hipertensión arterial (HTA) & 12 & $35.3 \% \%$ \\
\hline HTA e hipotiroidismo & 8 & $23.5 \%$ \\
\hline Hipotiroidismo & 3 & $8.8 \%$ \\
\hline $\begin{array}{l}\text { Otras enfermedades (artrosis, } \\
\text { osteoporosis) }\end{array}$ & 11 & $32.4 \%$ \\
\hline \multicolumn{3}{|l|}{ Antidiabéticos usados } \\
\hline Metformina & 29 & $50.88 \%$ \\
\hline Metformina+Glibenclamida & 6 & $10.53 \%$ \\
\hline $\begin{array}{l}\text { Metformina+iDPP-4 (sitagliptina - } \\
\text { linagliptina)* }\end{array}$ & 7 & $12.28 \%$ \\
\hline Metformina+insulina, o insulina sola ${ }^{\circ}$ & 9 & $15.78 \%$ \\
\hline $\begin{array}{l}\text { Metformina+Glimepirida, o glime- } \\
\text { pirida sola }\end{array}$ & 2 & $3.51 \%$ \\
\hline Desconocen/no recuerdan & 4 & $7.02 \%$ \\
\hline
\end{tabular}

Fuente: Elaborado por los autores
Para identificar posible asociación estadística entre variables se transformó la variable "estados de salud" en dicotómica; donde el código 11111 equivale a la categoría "sin problemas de salud" y los demás códigos se equipararon a la categoría "con problemas de salud". Al buscar su asociación mediante la prueba de chi cuadrado con las variables sexo, instrucción, estado civil, presencia de otras enfermedades, tipo de trabajo, salario y número de miembros del hogar que perciben los ingresos económicos, sólo se identificó asociación estadística con esta última variable (Tabla 5).

Tabla 2 - Variables económicas en los participantes

$\begin{array}{ll}\text { Variable } & \text { Frecuencia Porcentaje } \\ \text { Trabajo } & \end{array}$

$\begin{array}{lll}\text { No trabaja } & 38 & 66.7 \% \\ \text { Privado } & 9 & 15.8 \% \\ \text { Público } & 1 & 1.8 \% \\ \text { Otro } & 9 & 15.8 \%\end{array}$

Ingresos económi$\cos$

Menos de 375 US\$*

$\begin{array}{ll}28 & 49.1 \% \\ 16 & 28.1 \% \\ 8 & 14 \% \\ 5 & 8.8 \%\end{array}$

Otro valor $8.8 \%$

\section{Miembros del hogar} que perciben ingresos

$\begin{array}{lll}1 \text { miembro } & 36 & 63.2 \% \\ 2 \text { miembros } & 13 & 22.8 \% \\ 3 \text { o más } & 8 & 14 \%\end{array}$

* Para el año de aprobación de este estudio (2017), el salario básico en Ecuador era este valor.

Fuente: Elaborado por los autores

Dado que las variables salud en el día de la entrevista (determinado mediante la EVA) y edad se encuentran medidas de manera cuantitativa, se buscó asociación estadística entre ellas mediante una prueba t, encontrándose asociación con significancia estadística (t: 53.924, $\mathrm{p}<0.001$, IC al $95 \%$ 65.254-70.290). Se calculó también el coeficiente de correlación de Pearson el cual también tuvo significancia estadística (r: -0.292, p:0.027) aunque en un nivel débil. Además, se calculó el coeficiente de determinación $\left(r^{2}\right)$ cuyo valor fue de 0.085; es decir el $8.5 \%$ de la calidad de vida expresada mediante la EVA se debería a la edad. 


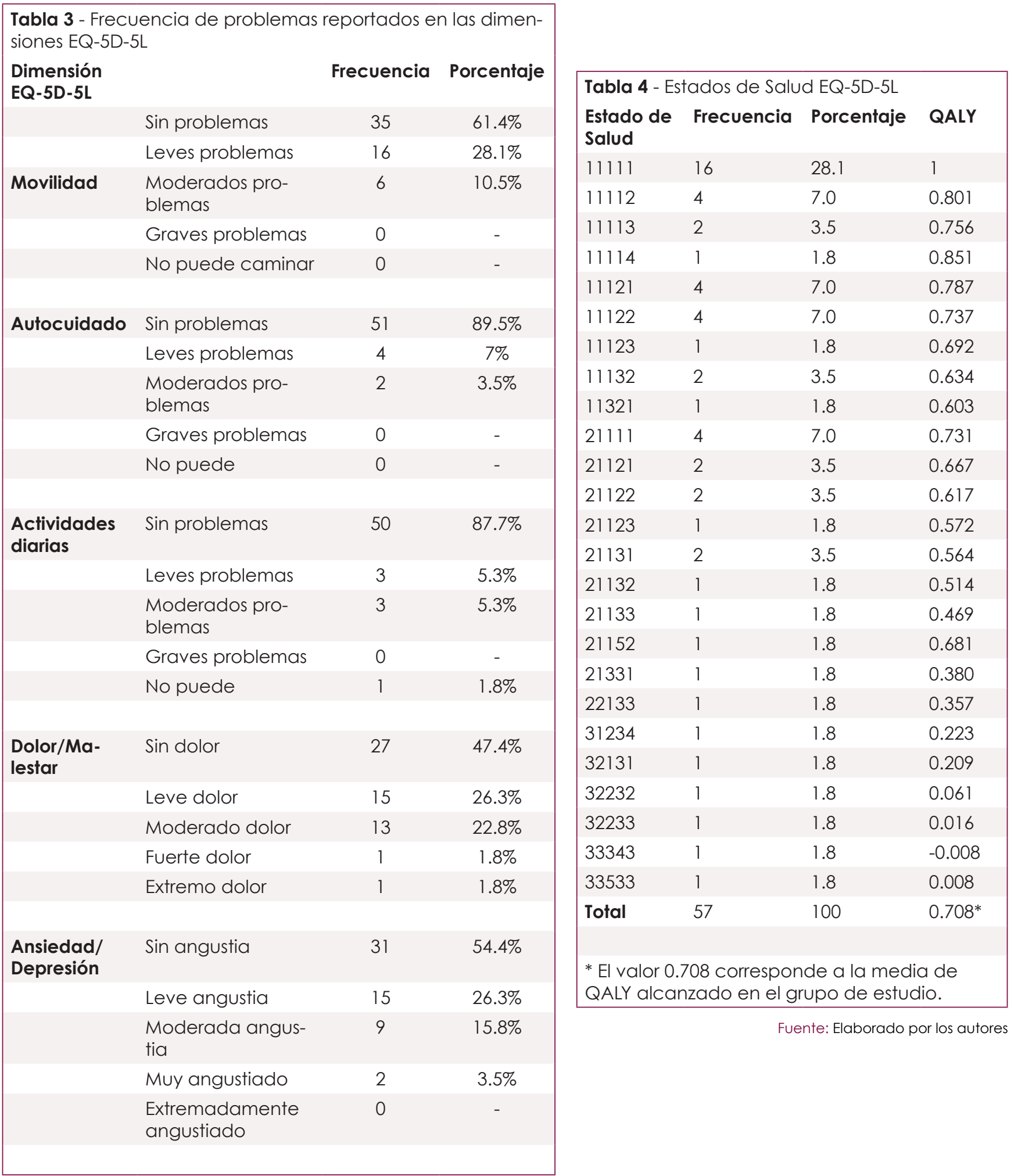




$\begin{array}{lcc}\text { Tabla } 5 \text { - Asociación estadística entre Estados de Salud y las demás variables cualitativas estudiadas } & \\ & \text { Chi cuadrado } & \mathbf{p} \\ \text { Variable } & 0.04 & 0.948 \\ \text { Sexo } & 6.599 & 0.159 \\ \text { Instrucción } & 1.400 & 0.706 \\ \text { Estado civil } & 0.075 & 0.784 \\ \text { Presencia de otras enfermedades } & 0.681 & 0.878 \\ \text { Tipo de trabajo } & 7.251 & 0.064 \\ \text { Salario } & 14.103 & 0.001 \\ \text { Miembros del hogar que perciben ingresos } & & \end{array}$

\section{Discusión}

En el grupo estudiado conformado principalmente por mujeres mayores de 65 años en promedio, metformina fue el antidiabético más utilizado por los pacientes, seguido por la combinación entre metformina e insulina o insulina sola, y en tercer lugar la asociación metformina e iDPP-4 (sitagliptina con mayor frecuencia). Además, destaca que el $66.7 \%$ de encuestados refirió no trabajar y, el $49.1 \%$ mencionó recibir ingresos (en dólares americanos) menores al equivalente a un salario básico de Ecuador para el año de aprobación del estudio (375 US\$). En cuanto a las dimensiones de calidad de vida evaluadas, menos de la tercera parte de pacientes refirió tener un estado de salud perfecto en las cinco dimensiones estudiadas (28.1\%), con una aparente asociación al número de miembros del hogar que reciben ingresos económicos. En tanto que el valor de la EVA

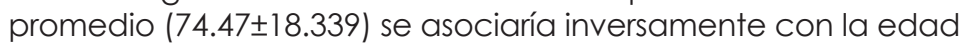
de los participantes, pero de manera débil. Con estos datos se obtuvo una utilidad media de 0.708QALY.

Son pocos los estudios reportados a nivel regional respecto a la calidad de vida en pacientes con diabetes y sus factores asociados; existiendo amplia heterogeneidad en cuanto a las características de estos, niveles de atención, y con tamaños muestrales muy diferentes al estudio aquí realizado, lo que dificulta las respectivas comparaciones. La mayor evidencia corresponde a Norteamérica, países europeos y asiáticos; lo cual puede influir en el contraste de información por el contexto de cada uno de ellos.

Varios estudios en el contexto latinoamericano y mundial reportan una media de edad de los pacientes evaluados superior a los 60 años, similar a lo identificado en este estudio. (19-21) Otras experiencias muestran medias de edad inferiores a los 60 años (22,23), e inclusive los estudios realizados en España por Collado y colaboradores y en India por Parikh y Patel $(24,25)$ utilizaron grupos etarios a partir de los 18 años; sin embargo, la mayor cantidad de participantes en estos estudios (alrededor del 50\% o más) correspondía a individuos de más de 60 años. En una experiencia realizada en Brasil por Tonetto y colaboradores (26) se mostró que conforme aumentaba el nivel de complejidad de la unidad de salud que atendía a los pacientes su edad se incrementaba, siendo la unidad de atención de salud de segundo nivel la que mostró la media de edad más alta (63.95 años) en comparación con las unidades de primero y tercer nivel, lo cual es concordante con lo encontrado en este estudio.
En relación al sexo, estudios realizados en Reino Unido (22), Navarra-España(19), Paraguay (23), Canadá (21) y Brasil (26), reportan un mayor número de participantes de sexo masculino; mientras que datos similares a esta investigación donde predomina el sexo femenino se reportaron en una investigación a nivel de 29 centros de atención primaria españoles ${ }^{(20)}$, en otra en Perú (27), así como en un estudio realizado en la India (proporción de mujeres del 60.3\%) ${ }^{(25)}$. Tomando como referencia los datos de la Encuesta Nacional de Salud y Nutrición - ENSANUT - realizada en Ecuador (17), se encuentra que en el grupo de edad de 50 a 59 años el porcentaje de individuos con diabetes mellitus tipo 2 corresponde en un $65.67 \%$ al sexo femenino y menciona que pasados los 60 años estos valores deberían aumentar (dato no reportado en ese estudio).

En cuanto al estado civil, los datos son similares a lo reportado por Brítez y Torres en su estudio en Paraguay (64.36\%) ${ }^{(23)}$ y por Oliveros y colaboradores en su experiencia en Perú (70.3\%) (27); mientras que fue algo superior a lo reportado en las unidades de salud de segundo nivel del estudio brasileño (40\%) (26). Considerando lo reportado en el nivel de escolaridad; tenemos datos similares a lo reportado por Oliveros y colaboradores. En el estudio realizado en Paraguay la escolaridad predominante fue la secundaria.

El estudio en India de Parikh y Patel ${ }^{(25)}$ reportó que el $58.1 \%$ de sus 358 participantes presentó alguna comorbilidad adicional, mientras que en el estudio realizado en 888 pacientes atendidos en consultas de atención primaria en España ${ }^{(20)}$ más del $50 \%$ de participantes presentó alguna comorbilidad, destacándose la Hipertensión Arterial, Hiperlipemia y Síndrome de resistencia a la Insulina. En este estudio los resultados fueron similares, pues más de la mitad de sus participantes presentó alguna comorbilidad, predominando la Hipertensión Arterial y el Hipotiroidismo. 
Sin que se especifique el antidiabético oral más usado; en los estudios de Brítez y Torres (23), Matza y colaboradores (22) y Mata Cases y colaboradores ${ }^{(20)}$ fueron los antidiabéticos orales los medicamentos más utilizados por los pacientes evaluados similar a lo que reporta esta investigación.

En relación con si el paciente tiene empleo o no, un estudio realizado en Reino Unido con 25 participantes ${ }^{(22)}$ evidenció resultados opuestos a esta investigación, pues el $44 \%$ de sus participantes refirió tener un empleo a tiempo completo. En los 53 participantes del estudio realizado por Tonetto y colaboradores en la ciudad de Ribeirão Preto-Brasil (26) el $47.4 \%$ refirió ser jubilado/pensionista y el $36.8 \%$ trabajador activo, con apenas un $10.5 \%$ de participantes desempleados. La investigación realizada en Lima-Perú por Oliveros y colaboradores en 330 pacientes (27) encontró que el $55.8 \%$ de sus participantes estaba desempleado, y que el $51.5 \%$ de los estudiados tenía ingresos económicos entre US\$232y US\$ 464. En ninguno de estos estudios se especificó el número de miembros del hogar que perciben ingresos económicos.

Al contrastar los resultados alcanzados por los participantes en este estudio en las cinco dimensiones de calidad de vida evaluadas; se encuentran un valor menor al de la investigación de Collado y colaboradores (24) que con la participación de 1857 personas con diabetes mellitus tipo 2 de diferentes regiones de España reportó un $36.66 \%$ con estado de salud perfecto (código 11111), con la consideración de que estos datos corresponden a una población comprendida entre los 18 y 90 años o más. Además, en esta investigación la dimensión con el mayor efecto techo fue la de autocuidado $(76.4 \%)$ y la de menor efecto techo fue dolor/disconfort (45.6\%), coincidiendo en estas dimensiones a lo reportado en este estudio. Hervás y colaboradores ${ }^{(19)}$ en su estudio con 95 pacientes con diabetes mellitus tipo 2 en Navarra-España y con un promedio de edad similar a este estudio, coinciden en cuanto a la identificación de la dimensión de "autocuidado" como la de mayor efecto techo, seguidas en orden decreciente por las dimensiones actividades diarias, movilidad, ansiedad/depresión y finalmente dolor/malestar. El puntaje promedio en la EVA de dichos participantes fue 64.6, la cual es 9.87 puntos menor a la de este estudio.

La investigación en India de Parikh y Patel (25) realizada en 358 pacientes con diabetes mellitus tipo 2 en relación a los resultados del instrumento EQ-5D-5L y la EVA es muy coincidente con el presente estudio, pues mantiene a la dimensión autocuidado como la de mayor efecto techo sin compromiso de salud alguno (86.8\%) y al dolor/disconfort como la de menor efecto techo (46.6\%); además con una media en la EVA de 78,83 $\pm 15,02$ el cual es algo mayor al de este estudio $(74,47 \pm 18,34)$. El estudio realizado en Canadá por McClure y colaboradores en 1927 pacientes con diabetes mellitus tipo $2^{(21)}$ reporta un valor menor de participantes con un estado de salud perfecto (codificación 1111 1=15.9\%); sin embargo, la media obtenida en la EVA es un poco mayor $(79 \pm 17,1)$.

Al contrastar la calidad de vida reportada en nuestro estudio y expresada como AVAC-QALY, se muestra menor a los resultados obtenidos en estudios realizados en pacientes con diabetes tanto en España en el estudio de Collado y colaboradores (QALY promedio de 0.742 ) ${ }^{(24)}$, así como a los reportados en
Suecia por Kiadaliri y colaboradores (QALY promedio de 0.88) ${ }^{(28)}$. Debe mencionarse que los resultados españoles se obtuvieron con la aplicación del instrumento de EuroQol de 5 niveles (5L) y el sueco fue con el de 3 niveles (3L).

En el estudio de Mata Cases y colaboradores (20) se encontraron asociaciones entre una mejor cuantificación de la calidad de vida y variables como sexo (mejor en hombres), edad (mejor en pacientes más jóvenes), sin complicaciones asociadas a diabetes, no obesidad y sin tratamiento con insulina. Mientras que en la experiencia de Parikh y Patel (25), entre los escores obtenidos en el EQ-5D-5L y el valor obtenido en la EVA existió una asociación estadística negativa con las variables edad (menores escores a mayor edad), sexo (menor escores en varones), presencia de comorbilidades, y no control de la diabetes. En nuestro estudio apenas se encontró una aparente asociación de calidad de vida (expresada como EVA) con la edad de los participantes.

En cuanto a investigaciones realizadas a nivel local; se encontraron dos estudios realizados en Quito, concretamente en pacientes con diabetes mellitus tipo 2 domiciliados en el sur de la ciudad; correspondiendo ambos a trabajos de titulación de grado y postgrado respectivamente. El primero de ellos ${ }^{(29)}$ evaluó 103 pacientes con diabetes mellitus tipo 2 con el instrumento EQ-5D-5L en los centros de salud de El Rocío y La Victoria. Los pacientes estudiados fueron principalmente mujeres $(76.2 \%)$, mayores de 50 años, con escolaridad primaria (50.5\%), con trabajo no remunerado del hogar (56.4\%), e ingresos menores a un salario básico unificado -SBU- (54.5\%), en tratamiento principalmente con antidiabéticos orales (80.2\%), con más de 1 año de evolución de la enfermedad (94.1\%), y al menos 1 comorbilidad (41.6\%). En ellos se encontró una media del QALY de 0.69, la cual es menor al grupo de nuestro estudio, siendo las dimensiones mejor evaluadas las de autocuidado y actividad. En cuanto al valor de la EVA, obtuvieron una moda de $70 \%$, valor inferior al de la presente experiencia. Los valores más altos de calidad de vida se asociaron positivamente principalmente con el control glicémico medido con $\mathrm{HbAlc}$, tratamiento con antidiabéticos orales, ausencia de comorbilidades, enfermedad con evolución menor o igual a 5 años, ingresos superiores a 1 salario básico y el vivir en familias consideradas funcionales con el instrumento APGAR familiar; mientras que en nuestro estudio existió una aparente asociación entre los niveles de calidad de vida, la edad de los participantes y el número de individuos que perciben ingresos en el hogar.

En la experiencia de Espinosa y Romero (30), evaluaron a 325 pacientes durante el año 2016 
en el Centro de Salud de Chimbacalle en Quito. Participaron principalmente mujeres (80.6\%), mayores a 65 años (52.9\%), con menos de 10 años con diabetes mellitus tipo 2 (57.5\%), principalmente en tratamiento con antidiabéticos orales (56\%). Para la cuantificación de calidad de vida utilizaron la versión del instrumento EuroQol de 3 dimensiones (EQ-5D-3L) a diferencia del presente estudio que usó la de 5 dimensiones: encontrando una media en el EQ-SCORE (QALY) de 0.84 y en la EVA de $80 \%$; valores superiores a los reportados en nuestro estudio. Los valores de calidad de vida se asociaron con el tiempo de evolución de la enfermedad, la presencia de hipertensión arterial y la edad de los pacientes; siendo sólo esta última asociación (edad) similar a lo presentado en nuestra investigación.

\section{Limitaciones}

Si bien estos datos de medidas de utilidad expresadas en QALY se muestran menores lo cual puede explicarse en parte por el algoritmo de cálculo, así como al poco número de individuos estudiados y su método de selección; constituyen los primeros datos de este tipo para una unidad de salud en Ecuador y que podrían emplearse en estudios de costo-utilidad de tecnologías sanitarias. Como limitaciones del estudio; la inclusión en el estudio de pacientes con diabetes sin complicaciones mayores puede explicar el nivel de calidad de vida obtenido en los resultados, ya que "a priori" pudieron ser individuos que desde un principio tendrían mejor apreciación de ella. También la aparente poca asociación de la calidad de vida con otros factores socioeconómicos podría relacionarse con el pequeño número de participantes y estrictos criterios de exclusión, por lo que es recomendable una nueva experiencia con un mayor número de individuos y en diferentes unidades de salud. Otro factor no evaluado en este estudio y que podría modificar la calidad de vida, es el tiempo de duración de la enfermedad en los pacientes estudiados.

Como potenciales investigaciones derivadas de esta experiencia, podrían estudiarse la calidad de vida de los pacientes con diabetes mellitus tipo 2 de acuerdo con el tratamiento recibi- do (ej. insulinoterapia versus otros tratamientos), su relación con el tiempo de evolución de la enfermedad, y si existe relación alguna entre el automonitoreo glucémico y la calidad de vida. Además, se recomienda realizar un estudio de validación del mencionado algoritmo de cálculo para Ecuador, y como en todo estudio transversal las estimaciones de asociación presentadas deben tomarse con cautela.

\section{Conclusiones}

Considerando el resultado global del escore EQ-5D-5L, los pacientes con diabetes mellitus tipo 2 participantes en este estudio reportan un aparente mejor nivel de calidad de vida en comparación a países con niveles económicos superiores, como es el caso de España (Navarra) y Canadá. En el análisis por dimensiones, los pacientes estudiados tienen un comportamiento similar a otros en diferentes contextos, en cuanto a la dimensión con mejor valoración en calidad de vida (autocuidado), y la con el menor valor (dolor/disconfort); así como promedios muy similares en los puntajes obtenidos en la escala visual analógica. Estos primeros datos servirán como línea de base para futuras experiencias en la unidad de salud, así como para la realización inicial de modelados matemáticos en temas de toma de decisiones basados en la costo-efectividad de intervenciones en este grupo de pacientes.

\section{Agradecimientos}

Al Dr. Édgar León Segovia, Director de Docencia del Hospital Un Canto a la Vida en el año 2018, por su apoyo para la realización de este estudio.

\section{Referencias}

1. Millán J, Palés J, Morán J. Principios de Educación Médica. Desde el grado hasta e desarrollo profesional. Primera ed. Madrid: Editorial Médica Panamericana; 2015. 732 p.

2. Davins Miralles J. Gestión de la calidad. In: Casado V, Calero S, Cordón F, Ezquerra M, García L, García G, et al., editors. Tratado de Medicina Familiar y Comunitaria Volumen I. Segunda ed. Barcelona: Editorial Médica Panamericana; 2012. p. 1283.

3. Reyes H. Introducción a la evaluación económica de tecnologías en salud. In: Ruíz Morales A, Gomez-Restrepo C, editors. Epdemiología Clínica Investigación Clínica Aplicada. Segunda ed. Bogotá: Editorial Médica Panamericana; 2015. p. 625.

4. Rascati KL. Essentials of Pharmacoecono mics. Second. Philadelphia: Lippincott Williams \& Wilkins; 2014. 1-295 p. 5rummond $M$, Sculpher $M$, Torrance $G$ 'Brien B, Stoddart G. Methods for the Economic Evaluation of Health Care Progam mes. Third. New York: Oxford University Press; 2005. 1-379 p.

6. Soto Álvarez J. Evaluación económica de medicamentos y tecnologías sanitarias: Principios, métodos y aplicaciones en política sanitaria. Primera ed. Springer, editor. Madrid; 2012. 1-328 p.

7. Ruiz Morales Á, Gómez-Restrepo C. Sección 5: Análisis Económicos y evaluación de la tecnología. In: Epidemiología Clínica Investigación Clínica Aplicada. Segunda. Bogotá: Editorial Médica Panamericana; 2015. p. 643.

8. Instituto Nacional de Cardiologia-INC. Núcleo de Avaliação de Tecnologias em Saúde (NATS-INC) [Internet]. Estudo QALYBrasil. 2019 [citad0 2019 Dic 10]. Disponible en: http:// natsinc.org/wpress/euroqol/?page_id=767

9. van Reenen M, Janssen B. EQ-5D-5L User GUide - Basic information on how to use the EQ5D-5L instrument. Version 21. 2015; (Octubre).

10. Cabasés JM. El EQ-5D como medida de resultados en salud The EQ-5D as a measure of health outcomes. Gac Sanit. 2015:29(6):4013.

11. Kind $P$, Leese $B$, Hardman $G$. Demonstrating the value of EQ-5D as a tool for monitoring health status in primary care. 1997;

2. Herdman, M: Badia, X: Berra S. El EuroQol-5D: una alternativa sencilla para la medición de la calidad de vida relacionada con la salud en Atención Primaria. Aten Primaria. 2001;28(6):425-9. 
13. Grandy S, Fox KM. EQ-5D visual analog scale and utility index values in individuals with diabetes and at risk for diabetes: Findings from the Study to Help Improve Early evaluation and management of risk factors Leading to Diabetes (SHIELD). Health Qual Life Outcomes [Internet]. 2008;6:18. Disponible en: mes [Internet]. 2008;6:18. Disponible en: render.fcgie artid=2266905\&tool=pmcentrez\& render.fcgi? artid $=226696$
rendertype $=$ abstract

14. Pan CW, Sun HP, Wang $X, M a Q, X \cup Y$, Luo $N$ et al. The EQ-5D-5L index score is more discriminative than the EQ-5D-3L index score in diabetes patients. Qual Life Res [Internet]. 2014:1767-74. Disponible en: http://www. 2014;1767-74. Disponible en: http://

15. Mulhern B, Meadows K. The construct validity and responsiveness of the EQ-5D, SF-6D and Diabetes Health Profile-18 in type 2 diabetes. Health Qual Life Outcomes [Internet]. 2014;12(1):42. Disponible en: http://www. ncbi.nlm.nih.gov/pubmed/24661350

16. Vandenbroucke JP, Von Elm E, Altman DG Gøtzsche PC, Mulrow CD, Pocock SJ, et al. Strengthening the Reporting of Observational Studies in Epidemiology (STROBE): Explanation and elaboration. Epidemiology. 2007;18(6):805-35.

17. Freire $\mathrm{W}$, Belmont $\mathrm{P}$, Gómez $\mathrm{L}$, Mendieta $\mathrm{M}$, Monge $R$, Piñeiros $P$, et al. Encuesta Nacional de Salud y Nutrición. ENSANUT-ECU 2012. Tomo I. 2014. 718 p.

18. Santos $M$, Cintra MACT, Monteiro AL, Santos B, Gusmão-filho F, Andrade MV, et al. Brazilian Valuation of EQ-5D-3L Health States. Med Decis Mak [Internet]. 2016 Feb 22:36(2):253-63. Disponible en: http://journals.sagepub.com/ doi/10.1177/0272989X15613521
19. Hervás A, Zabaleta A, De Miguel G, Beldarrain O, Díez J. Calidad de vida relacionada con la salud en pacientes con diabetes mellitus tipo 2. An Sist Sanit Navar. 2007;30(1):45-52.

20. Mata Cases M Roset Gamisans M, Badia Llach X, Antoñanzas Villar F, Ragel Alcázar J. [Effect of type-2 diabetes mellitus on the quality of life of patients treated at primary care consultations in Spain]. Aten primaria [Internet]. 2003;31 (8):493-9. Disponible en: http:// www.ncbi.nlm.nih.gov/pubmed/12765587

21. McClure NS, Sayah F Al, Ohinmaa A, Johnson JA. Minimally Important Difference of the EQ5D-5L Index Score in Adults with Type 2 Diabe5D-5L Index Score in Adults with Type 2 Diabe-
tes. Value Heal [Internet]. 2018;21 (9):1090-7. Disponible en: http://dx.doi.org/10.1016/j. jval.2018.02.007

22. Matza LS, Boye KS, Stewart KD, Curtis BH, Reaney $M$. Landrian AS. A qualitative examination of the content validity of the EQ-5D-5L in patients with type 2 diabetes. Health Qual in patients with type 2 diabetes. Health Qual
Life Outcomes [Internet]. 2015;13(1):1-10. DisLonible en: http://dx.doi.org/10.1186/s12955015-0373-7

23. Brítez M, Torres de Taboada E. Quality of life in patients with type 2 diabetes mellitus. Del Nac. 2017:9(1):78-91.

24. Collado D, García M, Olivares PR, Adsuar JC. Datos normativos del EQ-5D-5L en pacientes diabéticos de España. Nutr Hosp. 2015;32(4):1595-602.

25. Parikh P, Patel V. Health-related Quality of Life of Patients with Type 2 Diabetes Mellitus at A Tertiary Care Hospital in India Using EQ 5D 5L. Indian J Endocr Metab. 2019;(23):407-11.

26. Tonetto IF de A, Baptista MHB, Gomides D dos
S, Pace AE. Quality of life of people with diabetes mellitus. Rev da Esc Enferm. 2019;53:1-8.

27. Oliveros-Lijap L, Ávila-Espinoza P, Ulloa V, Bernabe-Ortiz A. Calidad de vida en pacientes con neuropatía diabética periférica: estudio tictio transversal en Lima, Peru. Acta Med Peru [Internet]. 2018;35(3):160-7. Disponible en: www.optum.com/optum-outcomes/whatwe-do/

28. Kiadaliri AA, Eliasson B, Gerdtham U-G. Does the choice of EQ-5D tariff matter? A comparison of the Swedish EQ-5D-3L index score with UK, US, Germany and Denmark among type 2 diabetes patients. Health Qual Life Outcomes [Internet]. 2015;13(1):145. Disponible en: mes [Internet]. 2015;13(1):145. Disponib/2
http://www.hqlo.com/content/13/1/145

29. Escobar MG (2017). Percepción de la calidad de vida y su relación con el control glicémico en pacientes con diabetes tipo 2 en dos centros de salud clel Sur de Quito CS La Victoria Centraly Centro de Salud el Rocí Disto 17D07 durante el año 2016. [Tesis para optar por el Título de Especialista en Medicina Familiar y Comunitaria]. Quito: Universidad Central del Ecuador, Instituto Superior de Investigación y Postgrado. Quito, 2017. [Consultado 20 de marzo 2021]. Disponible en http:/ www. dspace.uce.edu.ec/handle/25000/10321

30. Espinosa C, Romero F. Calidad de vida EQ5D-3L de acuerdo a factores demográficos y clínicos en pacientes con diabetes mellitus tipo 2 del centro de salud de Chimbacalle en el mes de junio-julio del 2016. [Tesis previa a la obtención del título de médico cirujanol. Quito: Pontificia Universidad Católica del Ecuador Facultad de Medicina, 2016. del Ecuador Faculad [Consultado 20 de marzo 2021]. Disponible en http://repositorio.puce.edu.ec/handle/22000/1390 